\title{
Sistema de baixo custo para monitoramento remoto da qualidade da água
}

A qualidade da água para consumo humano influencia diretamente no bem-estar dos seus consumidores. Doenças infectocontagiosas de veiculação hídrica surgem como problemas de saúde pública que poderiam ser evitados pela fiscalização contínua dos sistemas de abastecimento de água quanto ao atendimento dos padrões de potabilidade apresentados na Portaria de Consolidação no 5/2017 do Ministério da Saúde. Comunidades que residem em áreas mais afastadas dos centros urbanos e que fazem uso de águas advindas de sistemas alternativos de abastecimento, são muitas vezes excluídas de ações de fiscalização dos parâmetros de qualidade da água, geralmente pela dificuldade de acesso e de logística que há para coleta de amostras nesses locais. Os parâmetros pH e Turbidez, utilizados para controle da qualidade água de solução alternativa coletiva, informam sobre a presença de substâncias contaminantes que podem nesses locais. Os parâmetros pH e Turbidez, utilizados para controle da qualidade água de solução alternativa coletiva, informam sobre a presença de substâncias contaminantes que podem
oferecer risco à saúde humana. $\mathrm{O}$ uso de sistemas de monitoramento remoto para acompanhar as variações desses parâmetros apresenta-se como solução de controle qualitativo da água que abastece tais comunidades, permitindo a verificação em tempo real de eventuais alterações na qualidade da água. Este trabalho teve como objetivo desenvolver um dispositivo de baixo custo para monitorar em tempo real os parâmetros de pH, turbidez e temperatura da água. Para isso, foi criado um hardware, contendo componentes sensoriais, controladores e de alimentação, seguindo-se a programação do software em linguagem Arduino, similar à linguagem C. A calibração e validação dos sensores foram feitas mediante ensaios no Laboratório de Saneamento da Universidade Federal de Campina Grande (UFCG), utilizando pHmetro e turbidímetro. O sistema foi testado em um reservatório de água da UFCG e enviava os dados coletados em tempo real para a plataforma online ThingSpeak, que apresentava graficamente as informaç̃os sobre os parâmetros de interesse. Os resultados obtidos comprovaram a eficiência do sistema para monitoramento do $\mathrm{pH}$, turbidez e temperatura da água, permitindo que este venha a ser futuramente utilizado para maximizar o controle da qualidade da água em cisternas rurais, por exemplo.

Palavras-chave: Medição inteligente; $\mathrm{pH}$; Turbidez; Comunidades rurais.

\section{Low cost system for remote monitoring of water quality}

\begin{abstract}
The quality of water for human consumption directly influences the well-being of its consumers. Infectious diseases transmitted by water appear as public health problems that could be avoided by the continu hum ins con Communities residing in areas more distant from urban centers and that make use of water from alternative supply systems, are often excluded from inspection actions of water quality Communities residing in areas more distant from urban centers and that make use of water from alternative supply systems, are often excluded from inspection actions of water quality
parameters, generally due to the difficulty of access and logistics that exist for collecting samples in these places. The $\mathrm{pH}$ and Turbidity parameters, used to control the quality of water from a collective alternative solution, inform about the presence of contaminating substances that can pose a risk to human health. The use of remote monitoring systems to monitor variation in these parameters is a solution for the qualitative control of the water that supplies these communities, allowing real-time verification of any changes in water quality. This work aimed to develop a low-cost device to monitor in real time the parameters of $\mathrm{pH}$, turbidity and water temperature. For this, hardware was created, containing sensory, controller and powe components, followed by programming the software in Arduino language, similar to the Clanguage. Of Campina Grande (UFCG), using pH meter and turbidimeter. The system was tested in a UFCG water reservoir and sent the collected data in real time to the online platform ThingSpeak, which graphically presented information about the parameters of interest. The results obtain w proved the efficiency of the system for monitoring the pH, tubidity and temperture of the water, allowing it to be used in the future to maximize the control of water aualty in 作 rural cisterns, for example.
\end{abstract}

Keywords: Smart metering; pH; Turbidity; Rural communities.

Topic: Tecnologia, Modelagem e Geoprocessamento

Reviewed anonymously in the process of blind peer.
Received: 05/06/2021

Approved: 26/06/2021
Igor Furtado Targino

Universidade Federal de Campina Grande, Brasil

http://lattes.cnpq.br/3687832975270058

igorfurtadot@gmail.com

Patricia Herminio Cunha Feitosa (1)

Universidade Federal de Campina Grande, Brasil

http://lattes.cnpq.br/8276706034302451

http://orcid.org/0000-0001-6937-0817

phcfeitosa@outlook.com

Dayse Luna Barbosa (iD

Universidade Federal de Campina Grande, Brasil

http://lattes.cnpq.br/7376198442355112

http://orcid.org/0000-0002-3209-270X

dayseluna@yahoo.com.br

d

DOI: 10.6008/CBPC2179-6858.2021.006.0055
Igor Antônio de Paiva Brandão (ic)

Universidade Federal de Campina Grande, Brasi

http://lattes.cnpq.br/0917850140413106

http://orcid.org/0000-0003-1562-4361

igorantonio.eng@gmail.com
Referencing this:

TARGINO, I. F.; FEITOSA, P. H. C.; BARBOSA, D. L.; BRANDÃO, I. A. P.. Sistema de baixo custo para monitoramento remoto da qualidade da água. Revista Ibero Americana de Ciências Ambientais, v.12, n.6, p.665-680, 2021. DOI: http://doi.org/10.6008/CBPC2179$\underline{6858.2021 .006 .0055}$ 


\section{INTRODUÇÃO}

A gestão dos processos de tratamento, distribuição e controle da qualidade da água deve ser feita tanto em sistemas de abastecimento de água convencionais, que contam com estações de tratamento e redes de distribuição, como nos sistemas alternativos de abastecimento de água que suprem as necessidades das pequenas comunidades, de forma a maximizar a qualidade de vida das pessoas de forma universal e equitativa (MACHADO et al., 2016). Entretanto, na ausência de sistemas de distribuição ou de soluções alternativas que atendam às normas vigentes de potabilidade da água, muitas pessoas ainda se sentem obrigadas a fazer o uso de águas sem nenhum tipo de controle qualitativo, além de muitas dessas não receberem o mínimo de instruções a respeito dos riscos que tal situação pode oferecer (SALLA et al., 2019).

Para uniformizar a qualidade da água que é distribuída às populações, suportes legais e normativos de padrões de potabilidade da água para consumo humano são desenvolvidos em todo o mundo, os quais são geralmente fiscalizados por intermédio dos órgãos de vigilância sanitária locais e pelos responsáveis pelo abastecimento da água. Tais normas estabelecem parâmetros para controle microbiológico, turbidez, substâncias químicas, substâncias radioativas, desinfecção, entre outros, além de instituir processos ideais para assegurar a eficiência no tratamento e distribuição da água, variando com a legislação de cada país (VENTURA et al., 2019).

Sistemas alternativos de abastecimento de água, como poços, cisternas e caminhões-pipa, são amplamente utilizados no nordeste brasileiro, principalmente nas zonas rurais, que muitas vezes não contam com ligações às redes públicas de fornecimento de água. Para a realização do monitoramento qualitativo da água nesses sistemas, custos com deslocamentos periódicos de equipes se fazem necessários. Diante disso, comumente a água proveniente de sistemas alternativos de abastecimento carece de vigilância e controle da sua qualidade (GOMES et al., 2016).

O monitoramento convencional da qualidade da água, realizado através do recolhimento de amostras e da realização de testes em laboratórios, é a forma mais comum para avaliação de parâmetros de segurança que uma água fornece quanto ao consumo humano. Entretanto, tecnologias têm sido desenvolvidas para minimizar o tempo de coleta e análise de amostras, contando com sensores para medição de propriedades da água e ferramentas computacionais para validação do modelo criado (ROCHA et al., 2018). Segundo Pasika et al. (2020), smart water quality monitoring (SWQS), ou monitoramento inteligente da qualidade da água, envolve sistemas compostos por microcontroladores e sensores, montados de forma compacta para verificação de parâmetros como pH, turbidez e temperatura, funcionando continuamente e enviando dados em tempo real para estações de monitoramento via tecnologias wireless. Aplicações como ThingSpeak e Temboo são comumente usadas como interface de interpretação e apresentação dos dados recebidos, podendo ser usadas em computadores ou dispositivos móveis.

O uso de sistemas de automação se mostra então como alternativa para auxiliar nas medições de parâmetros qualitativos da água, reduzindo esforços, custos e tempo de resposta ao permitir o acompanhamento de alterações nos parâmetros qualitativos da água em tempo real. Diante disso, este 
trabalho teve como objetivo o desenvolvimento de um protótipo de baixo custo para monitoramento da qualidade da água, mediante a vigilância dos parâmetros pH, turbidez e temperatura, assim como a avaliação de sua viabilidade para aplicações futuras em zonas rurais ou de difíceis acessos.

\section{METODOLOGIA}

O desenvolvimento do sistema inteligente para medição remota partiu da criação e montagem dos componentes de hardware e de software para monitoramento qualitativo da água. Os sensores foram inseridos dentro de um reservatório contendo água da torneira, proveniente do sistema convencional de abastecimento da cidade de Campina Grande, de forma que os mesmos ficassem em contato com a água. $\mathrm{O}$ reservatório foi mantido fechado com tampa durante todo o período de análise, sobre uma bancada de laboratório e sem contato direto com o sol. A Figura 1 detalha o esquema que foi usado para desenvolvimento do sistema inteligente.

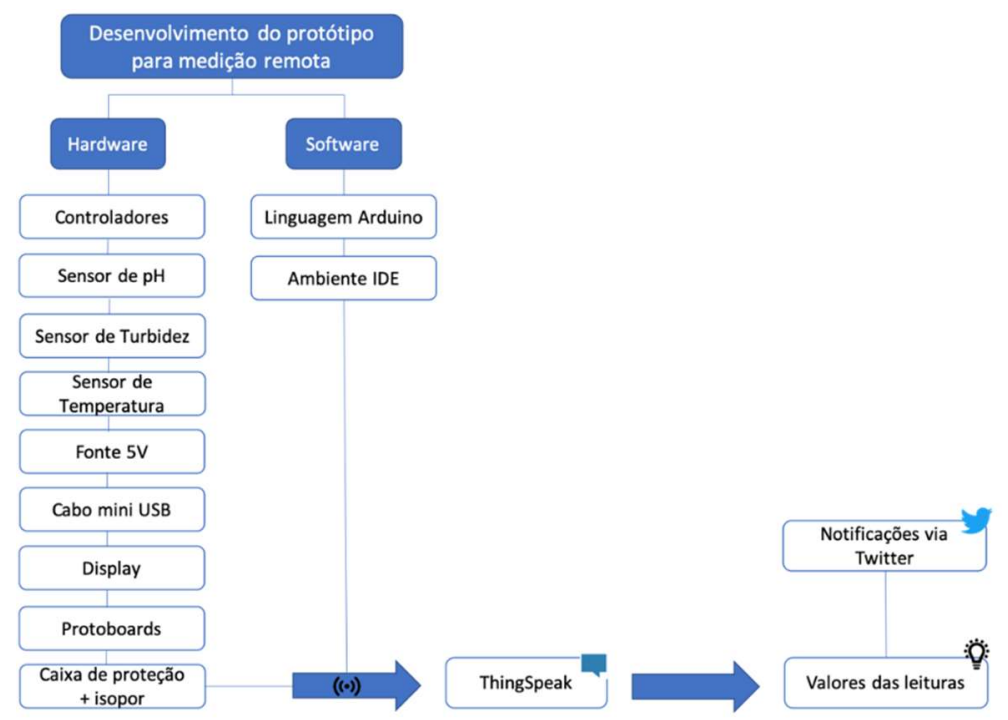

Figura 1: Esquema para desenvolvimento do sistema inteligente

Todos os testes foram realizados no laboratório de Saneamento da Universidade Federal de Campina Grande (UFCG). O módulo controlador conectava-se aos sensores através de cabos. Os equipamentos eletrônicos foram envolvidos por uma caixa de proteção, flutuando dentro do reservatório com a utilização de uma estrutura de isopor. Estando o sistema ligado a uma fonte de $5 \mathrm{~V}$ conectada à tomada, as rotinas de leituras dos parâmetros de qualidade em monitoramento tinham início.

O microcontrolador Arduino iniciava a rotina de medições com a leitura do pH da água, seguindo-se a medição da temperatura e depois da turbidez. Os dados das leituras de pH, temperatura e turbidez, captados na forma de pulsos elétricos pelas portas analógicas do microcontrolador, eram convertidos para valores reais de ambos os parâmetros, a partir da programação que foi instalada no microcontrolador. Realizadas duzentas medições de cada um dos parâmetros, os resultados das médias das leituras eram apresentados na tela OLED, conectada também ao Arduino.

Em seguida, os dados das leituras realizadas no Arduino eram enviados para o microcontrolador NodeMcu, que tinha a função de se conectar a uma rede Wi-Fi e enviar os dados para a plataforma 
ThingSpeak. O envio dos dados para o ThingSpeak foi possível mediante o cadastro de uma chave, fornecida por esta plataforma, na programação do software para monitoramento da qualidade da água. A escolha quanto a utilização do ThingSpeak se deu pela facilidade, gratuidade e praticidade quanto ao seu uso em diversos tipos de dispositivos móveis, permitindo ainda a análise gráfica de dados que são atualizados em tempo real.

O armazenamento e visualização dos dados ocorreu na plataforma ThingSpeak, onde os dados das leituras foram armazenados na nuvem e apresentados em forma de três gráficos, um para cada parâmetro analisado. Com o auxílio da ferramenta Matlab, foram criados histogramas dos dados de $\mathrm{pH}$ e turbidez, a fim de facilitar a visualização das entradas numéricas mais recorrentes. Também eram gerados gráficos que correlacionavam as variações de $\mathrm{pH}$ e turbidez com a temperatura. Enviados os dados para o ThingSpeak, o sistema entrava em modo de repouso por 30 minutos. Este intervalo de repouso foi estabelecido para poupar a vida útil da bateria do sistema, tendo também em vista a improvável variação dos parâmetros analisados em tão curto intervalo de tempo. Após os 30 minutos, o sistema era reiniciado automaticamente, conforme programado em seu software, refazendo toda a rotina de leitura e envio de dados.

Os gráficos de $\mathrm{pH}$, temperatura e turbidez foram atualizados em tempo real durante todo o tempo das medições. Cada entrada nova de dados informava o valor da leitura, a data e a hora da medição. A plataforma thingspeak permitia personalizar a escala de tempo no eixo $\mathrm{x}$ de cada gráfico, possibilitando a análise dos dados hora a hora ou ainda diariamente. Foi elaborada também uma programação no ThingSpeak que fazia publicações via Twitter a respeito do funcionamento do sistema, notificando o usuário em tempo real caso os valores de turbidez ou pH estivessem em desobediência aos valores citados na Portaria de Consolidação no5 de 2017 do Ministério da Saúde, apresentados na Tabela 1.

Tabela 1: Critérios e Valores de referência para o Monitoramento da Qualidade da água.

\begin{tabular}{|l|l|l|l|}
\hline \multicolumn{4}{|l|}{ Critérios e valores de referência para o Monitoramento da Qualidade da Água } \\
\hline $\mathrm{pH}$ & $\mathrm{pH}<6 \mathrm{e} \mathrm{pH}>9$ & Turbidez (NTU) \\
\hline Ruim & $6<=\mathrm{pH}<=9$ & Ruim & Turbidez $<5$ \\
\hline Bom & Bom & Turbidez $>=5$ \\
\hline
\end{tabular}

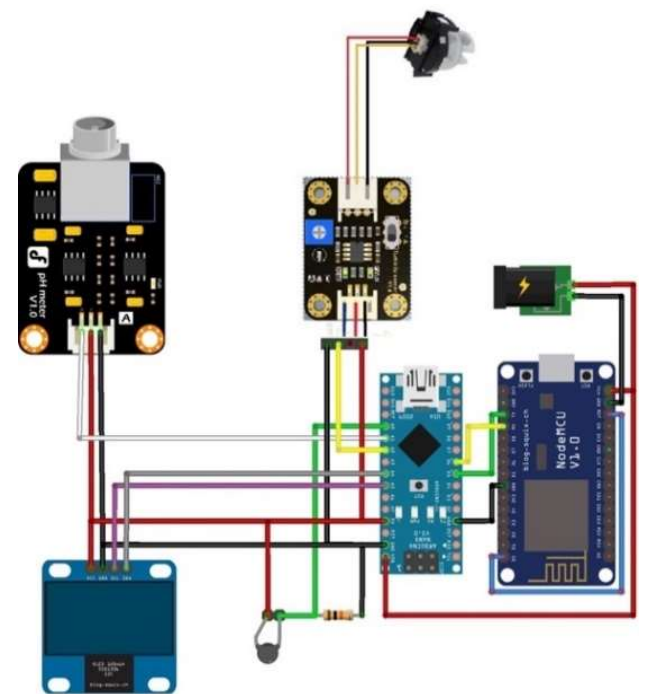

Figura 2: Esquema para montagem do hardware

A calibração e validação dos sensores de pH, temperatura e turbidez foram realizadas objetivando a 
minimização de possíveis erros e a maximização na exatidão dos resultados. O esquema de montagem do hardware está ilustrado na Figura 2.

O software utilizado no sistema deste estudo foi desenvolvido a partir do IDE do Arduino. As etapas de funcionamento do software são descritas no Quadro 1.

Quadro 1: Etapas de funcionamento do Software.

\begin{tabular}{|c|c|}
\hline $\begin{array}{l}\text { Etapa } \\
1\end{array}$ & Conexão do microcontrolador NodeMCU à Internet. \\
\hline $\begin{array}{l}\text { Etapa } \\
2\end{array}$ & Conexão do microcontrolador ao servidor NTP, do qual foram retirados os valores de data e hora. \\
\hline $\begin{array}{l}\text { Etapa } \\
3\end{array}$ & $\begin{array}{l}\text { Início da programação, em que foi ativada uma função para captura de pulsos elétricos pelos sensores sempre que estes } \\
\text { eram ativados, de } 30 \text { em } 30 \text { minutos. }\end{array}$ \\
\hline $\begin{array}{l}\text { Etapa } \\
4\end{array}$ & $\begin{array}{l}\text { Os valores numéricos fornecidos pelos sensores de } \mathrm{pH} \text {, temperatura e de turbidez foram convertidos para valores reais, } \\
\text { que podiam ser interpretados pelo usuário final. }\end{array}$ \\
\hline $\begin{array}{l}\text { Etapa } \\
5\end{array}$ & Os valores convertidos foram apresentados no display OLED. \\
\hline Etapa & $\begin{array}{l}\text { A cada intervalo de } 30 \text { minutos, os valores de } \mathrm{pH} \text {, temperatura e turbidez foram armazenados e enviados para o } \\
\text { ThingSpeak. O armazenamento local era zerado, permitindo assim que uma nova rotina de leitura fosse iniciada após o } \\
\text { intervalo de repouso. }\end{array}$ \\
\hline
\end{tabular}

O sensor de pH utilizado na montagem do sistema era formado por uma sonda com plug tipo BNC, que ficava em contado com a água, e um módulo para conexão com o microcontrolador Arduino, estando este contido dentro da caixa de proteção do sistema.

A calibração dos sensores de pH foi realizada com o auxílio de soluções tampão em laboratório. As soluções tampão utilizadas possuíam pH de 4, 7 e 10, representando, respectivamente, soluções básicas, neutras e ácidas. $\mathrm{O}$ sensor de $\mathrm{pH}$ foi conectado ao microcontrolador por meio de uma entrada analógica, que permitia a leitura de números que variavam de 0 a 1023. Cada leitura de pH era lida então pelo microntrolador como um número inteiro contido neste intervalo.

Com base nas faixas de valores de $\mathrm{pH}$ comumente encontradas para águas potáveis, foi estabelecido para este estudo a limitação da leitura de valores de pH entre 4 e 10 . A restrição da faixa de leitura do pH durante a calibração do sensor, permitiu a redução do percentual de erro obtido durante a conversão de valores lidos pelo microcontrolador para valores de $\mathrm{pH}$.

Para a conversão dos dados lidos para dados reais de $\mathrm{pH}$, foram elaboradas as seguintes equações de conversão:

$$
\begin{aligned}
& p H=[-0.02307692308 *(\text { valor da leitura })]+26.2 \\
& p H=[-0.01829268293 *(\text { valor da leitura })]+22.2195122
\end{aligned}
$$

A equação 1 foi utilizada na programação em Arduino quando os valores das leituras obtidas nos sensores fossem menores do que 832 , o que indicava que o pH real da solução estaria dentro do intervalo de 7 a 10. Caso o número obtido na leitura fosse maior ou igual a 832, a rotina de programação utilizaria a equação 2 para converter os números lidos em valores reais de $\mathrm{pH}$, estando estes contidos no intervalo de 4 a 7.

Para validação do sensor de $\mathrm{pH}$ utilizado neste estudo, foi utilizado um pHmetro de bancada $\mathrm{mPa}$ 210 do laboratório de saneamento da Universidade Federal de Campina Grande. O pHmetro do laboratório foi calibrado com as mesmas soluções tampão utilizadas na calibração do sistema desenvolvido. A Figura 3 
mostra a calibração do pHmetro do laboratório com soluções tampão, enquanto a Figura 4 mostra as leituras de $\mathrm{pH}$ em funcionamento.

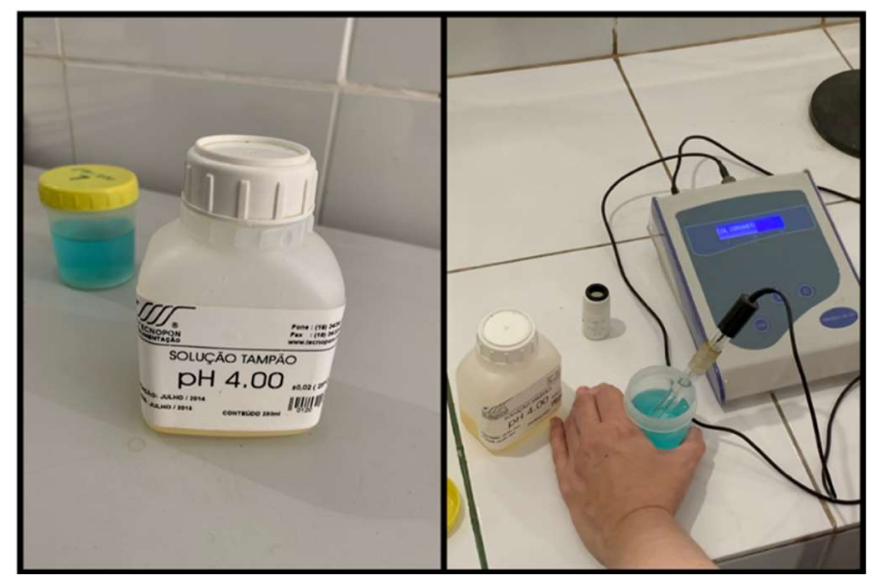

Figura 1: Calibração do pHmetro da UFCG.

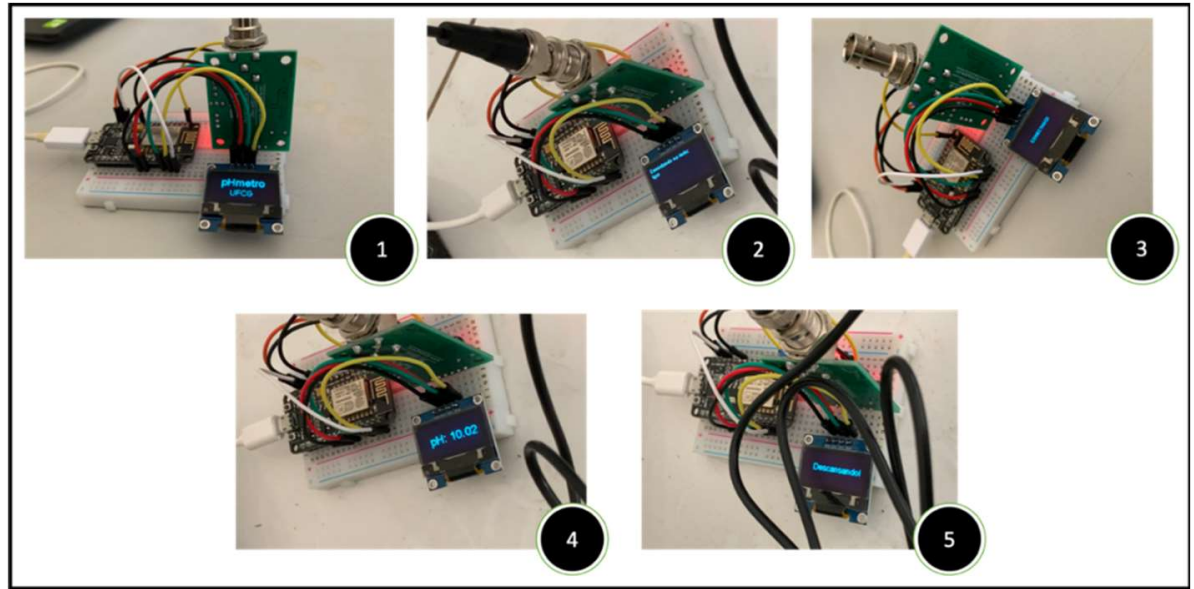

Figura 2: Leituras de pH com o protótipo desenvolvido.

O sensor de turbidez utilizado para a construção do sistema proposto neste trabalho, foi formado por uma sonda que emitia uma luz infravermelha, imperceptível à visão humana, capaz de detectar partículas que estivessem em suspensão na água e assim fazer a medição da taxa de dispersão de luz na água, além de um módulo para conexão com Arduino, este sem contato com a água. Para a calibração e validação do sensor de turbidez, foi utilizado um turbidímetro da marca Hach, modelo 2100P, pertencente ao laboratório de Saneamento da UFCG, conforme mostra a Figura 5.

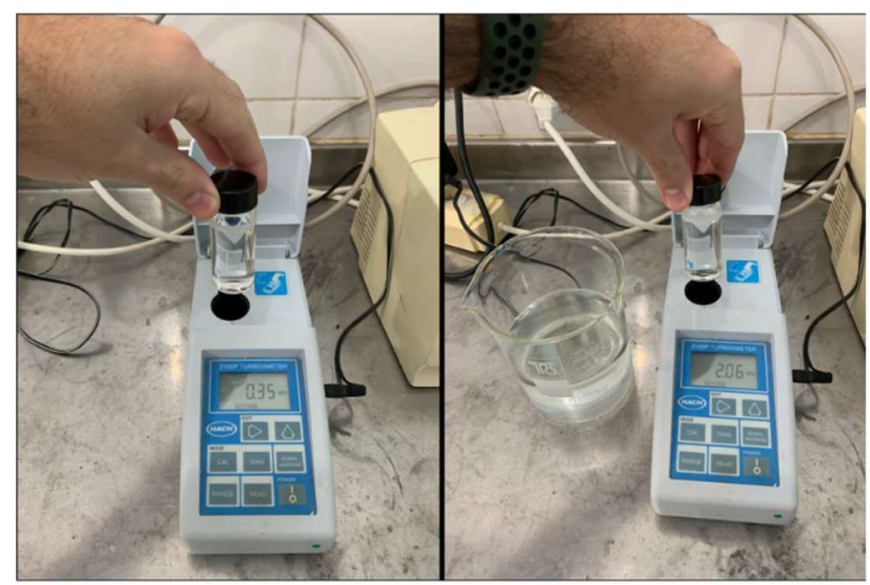

Figura 5: Validação da turbidez com turbidímetro Hach 2100P. 
O turbidímetro do laboratório foi calibrado com padrões de formazina estabilizada Stablcal, contidos em ampolas seladas com valores de 0,1, 20, 100 e 800 UNT. Após a calibração deste turbidímetro, uma amostra de água destilada com 0,35 UNT e uma mostra de água da torneira com 2,05 UNT foram utilizadas como valor de referência para a calibração do turbidímetro desenvolvido neste estudo. Para a obtenção dos valores de turbidez da água destilada e da água da torneira, utilizou-se o valor médio de quatro amostras de cada tipo de água.

Foi identificado que um valor de leitura analógica pelo microcontrolador com valor 213 equivalia à turbidez de 0,35 UNT. Um valor analógico de 225 equivalia a uma turbidez real de 2,05 UNT. Tais valores serviram como referência para a conversão de valores de tensão para valores reais de turbidez na IDE do Arduíno. Verificou-se que pequenas variações de tensão resultavam em grandes variações nos valores reais de turbidez. Tendo isso em vista, foi utilizado um potenciômetro de $1 \mathrm{~K} \Omega$ para regular com mais precisão os valores de tensão lidos pelo sensor de turbidez.

O monitoramento da temperatura da água foi feito por meio de um termômetro digital, do mesmo tipo que pode ser encontrado para o monitoramento da temperatura da água em aquários. Este era ligado a um resistor de $10 \mathrm{~K} \Omega$, que atuava como referência para o sensor de temperatura. Quando alguma variação de resistência elétrica em comparação com o resistor de $10 \mathrm{~K} \Omega$ era identificada, entendia que houve uma variação de temperatura, fornecendo a leitura em graus Celsius ao microcontrolador. Diferentemente dos sensores de pH e turbidez, nenhum módulo de processamento se fez necessário para intermediar a conexão entre o termômetro e o microcontrolador.

O tipo de conexão à internet foi testado neste trabalho de duas formas distintas: uma via 3G/4G e outra através de uma rede Wi-Fi. A conexão à internet 3G/4G foi feita a partir da instalação de um componente do tipo SIM800L acoplado ao microcontralador. Este componente permitia a inserção de um chip de telefonia móvel e garantia o acesso à internet em locais onde não existem redes Wi-Fi disponíveis. Esse tipo de conexão seria ideal para o monitoramento da qualidade da água em caminhões-pipa.

A fonte de energia utilizada para a alimentação do sistema também foi testada de duas formas. Inicialmente, testou-se a alimentação do sistema com uma bateria de $9 \mathrm{~V}$ alcalina, a fim de verificar a sua autonomia. Em seguida, utilizou-se uma fonte de $5 \mathrm{~V}$ e $1 \mathrm{~A}$ conectada à uma tomada 220V, que se conectava ao microcontrolador Arduino por meio de um cabo mini USB.

\section{RESULTADOS}

O período de captação dos dados foi de 11 dias, resultando em 344 leituras realizadas pelos sensores. As leituras de $\mathrm{pH}$, temperatura e turbidez eram enviadas para a plataforma ThingSpeak em intervalos de 30 minutos, conforme programado. Pequenos atrasos, na ordem de 25 segundos, foram identificados entre as medições armazenadas na nuvem, o que pode ser atribuído ao tempo que o NodeMcu levava para se conectar à rede $\mathrm{Wi}-\mathrm{Fi}$ local.

O tempo de estabilização médio para o sensor de pH passar a ler valores constantes em uma solução, foi de aproximadamente 3 minutos e 30 segundos. No primeiro dia de medições, os valores médios dos três 
parâmetros analisados neste trabalho apresentaram resultados que destoaram dos demais dias. Tal constatação pode ser atribuída ao fato de os valores terem sido captados apenas durante o período da tarde e noite, onde os valores de temperatura eram maiores.

Analisando as médias diárias de valores de $\mathrm{pH}$, foi possível identificar um valor médio de 7.42 no primeiro dia de medição. Os valores médios de $\mathrm{pH}$ foram tendo aumentos diários de 0.05 , até se estabilizarem em aproximadamente 7.65, até o final do período de 11 dias de captação dos dados. A Figura 6 mostra os valores médios de $\mathrm{pH}$ durante o período de análise.

Através da ferramenta Matlab, que operava como uma aplicação dentro da plataforma ThingSpeak, foi possível gerar também um histograma dos dados de leituras de $\mathrm{pH}$, facilitando a identificação dos valores mais recorrentes para este parâmetro. O Matlab funcionava com a escrita de um código, semelhante a linguagem $C$, tendo como inputs os dados armazenados na nuvem do ThingSpeak. $O$ gráfico de distribuição de frequências para dados de pH está apresentado na Figura 7, evidenciando a maior quantidade de leituras com valores entre 7,6 e 7,65.

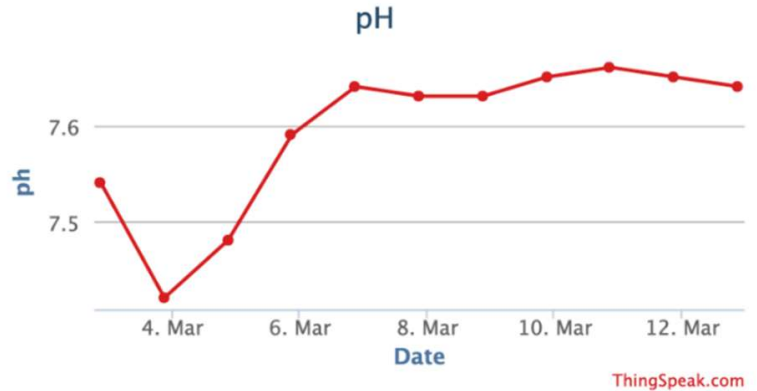

Figura 6: Valores diários das leituras de $\mathrm{pH}$.

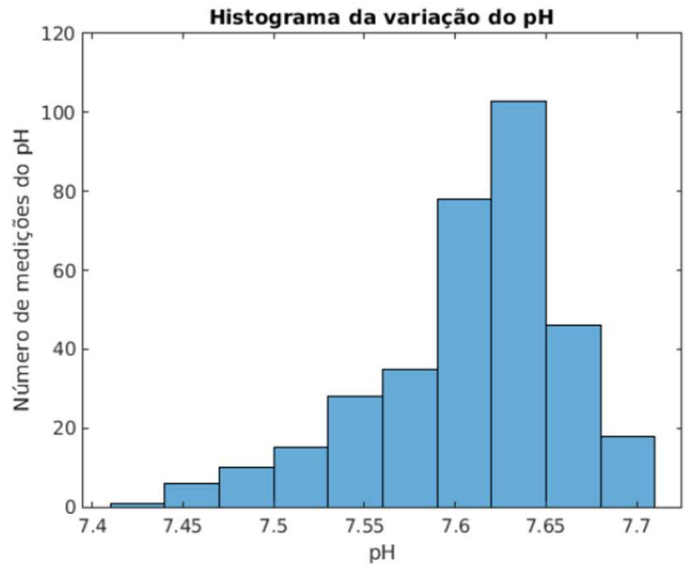

Figura 7: Histograma da variação do pH.

O Matlab também tornou possível correlacionar os dados de temperatura e pH mediante um gráfico, criado a partir da programação em linguagem própria desse software. Observando a Figura 8, é possível concluir que não há relevantes variações de pH com o aumento ou diminuição da temperatura. Os dados não se encontraram em posições próximas da linha de regressão ajustada, resultando em um valor de R-quadrado igual a 0,1047.

Como esperado, não houve significativas alterações no pH da água durante o período de avaliação do sistema, tendo em vista todos os testes terem sido realizados em ambiente controlado e fechado, longe de contaminações significativas.

Comparando os dados de $\mathrm{pH}$ lidos pelo sistema de monitoramento da qualidade da água com as leituras de uma amostra da mesma água realizadas no pHmetro do laboratório de saneamento da UFCG, no décimo primeiro dia de monitoramento, identificou-se uma diferença de $\pm 0,2$ entre as leituras dos dois equipamentos, equivalendo a um erro de $0,261 \%$. Para tal resultado, foi considerada a média de quatro medições do pH em ambos os pHmetros. Enquanto os valores variaram entre 7,60 a 7,65 no pHmetro no sistema de monitoramento, apresentaram resultados entre 7,65 e 7,85 no pHmetro do laboratório. 


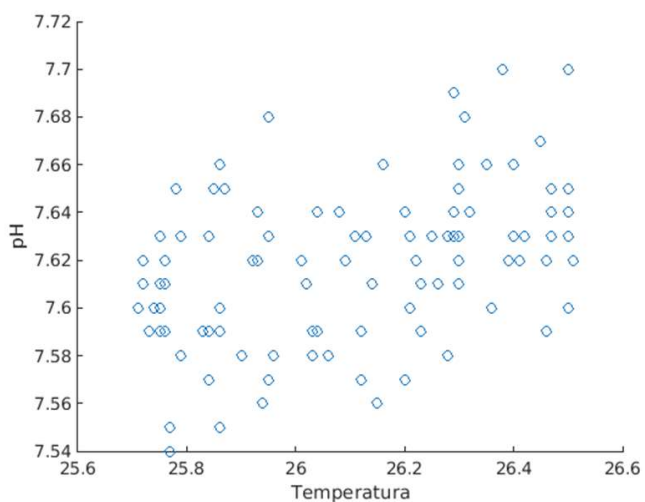

Figura 8: Gráfico de correlação entre pH e temperatura.
Turbidez

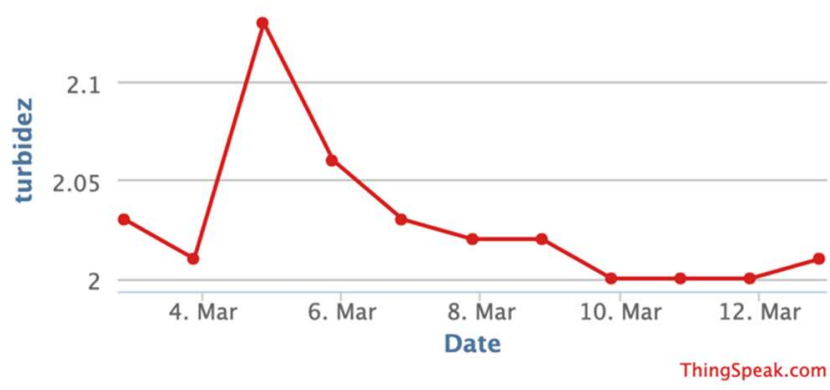

Figura 9: Valores diários das leituras de turbidez.

As notificações via Twitter deveriam acontecer caso os valores do $\mathrm{pH}$ fossem menores do que 6 ou maiores do que 9, o que não aconteceu durante o período de avaliação do sistema. Porém, em eventuais quedas de conexão à internet, o sistema emitiu notificações sobre a ausência da chegada de novos dados ao Thingspeak.

Analisando as leituras da turbidez, foi possível identificar uma sensibilidade do sensor a pequenas variações de temperatura. Ao longo do período de medições, a turbidez mostrou-se estável com médias diárias entre 2,00 UNT e 2,05 UNT. Porém, valores pontuais de até 2,80 UNT, nos horários menor temperatura, entre 09:30hrs e 10:30hrs, foram identificados. A Figura 9 mostra a variação das médias diárias da turbidez e a Figura 10 apresenta o histograma dos valores de turbidez, este desenvolvido a partir da ferramenta Matlab. A partir da Figura 10, é possível identificar a ocorrência de turbidez igual a 2 na grande maioria dos dados lidos.

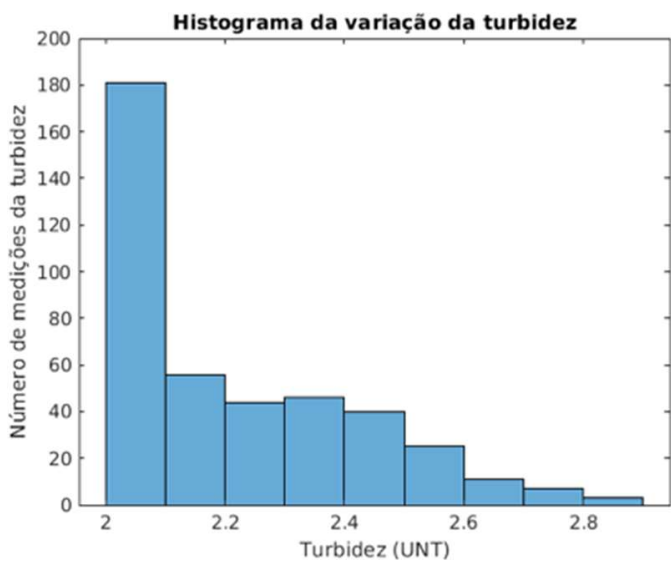

Figura 10: Histograma dos valores de turbidez.

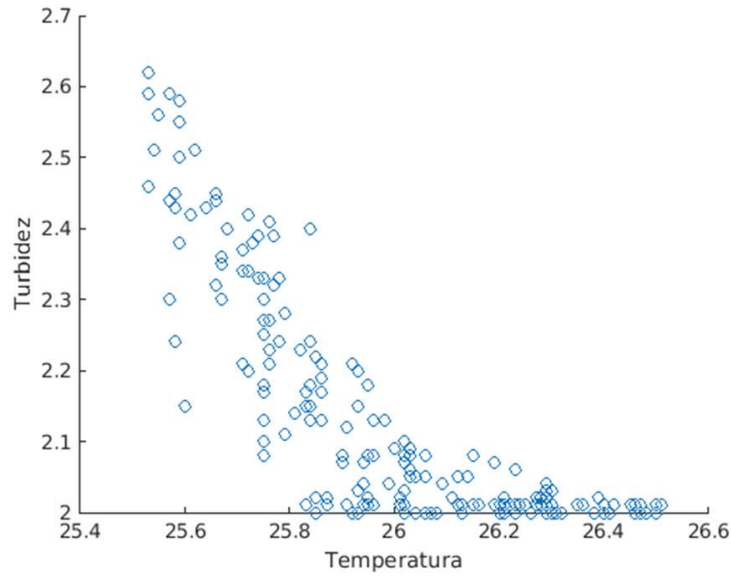

Figura 11: Gráfico de correlação entre turbidez e temperatura.

De acordo com Chagas (2015), os valores da turbidez estão diretamente relacionados com as substâncias que não estão dissolvidas na água, mas sim suspensas. Tendo isso em vista, as variações nos dados de turbidez lidos pelo sistema de monitoramento da qualidade da água podem estar relacionadas ao aumento da solubilidade dos sólidos suspensos na água provenientes do aumento da temperatura, resultando assim em uma diminuição da turvação (LAMBERTI, 2017). A Figura 11 mostra um gráfico de 
correlação entre a turbidez e a temperatura, evidenciando a tendência de a turbidez diminuir com o aumento da temperatura. O valor de R-quadrado para o modelo que correlaciona essas variáveis foi de 0,7782.

Devido ao uso do potenciômetro, utilizado para facilitar a calibração do sensor de turbidez, o valor mínimo da turbidez ficou limitado a 2,0 UNT. Porém, sempre que esse valor apareceu, ficou subentendido que o valor real lido poderia ser igual ou menor a 2,0 UNT. Para efeito de validação, utilizou-se o turbidímetro do laboratório de saneamento da UFCG. Neste, a partir de quatro medições da turbidez em uma amostra da mesma água utilizada para testar o sistema deste estudo, foi identificado um valor médio de 0,58 UNT no décimo dia de monitoramento, evidenciando tal limitação nas leituras mínimas captadas pelo sensor de turbidez deste estudo. Com isso, o erro apresentado pelo sistema desenvolvido com relação as leituras de turbidez foram de $2,45 \%$. Caso o valor da turbidez fosse maior ou igual a 5 , notificações via twitter seriam enviadas, possibilitando que, em situações práticas de monitoramento da qualidade da água, ações corretivas fossem tomadas.

\section{DISCUSSÃO}

Em todo o mundo, a turbidez é frequentemente utilizada como indicador da qualidade da água potável, pois esse parâmetro, embora por si só não represente risco à saúde humana, pode revelar a presença de contaminação da água por microrganismos patogênicos (STEVENSON et al., 2019). Além de apresentar aparência, sabor e cheiro desagradáveis, a água de beber com elevados índices de turbidez pode ser prejudicial à saúde humana. Muoio et al. (2020) concluíram que a probabilidade de contração de doenças gastrointestinais por meio do consumo da água de beber aumenta conforme maior for o nível de turbidez dessa.

Isso posto, caso a turbidez não seja removida, a água pode se tornar um meio propício ao crescimento de microrganismos patógenos (ABOUBARAKA et al., 2017). Portanto, a turbidez identificada na água analisada também se mostrou em conformidade com os padrões de potabilidade estabelecidos na Portaria de Consolidação no5 de 2017 do Ministério da Saúde, sempre apresentando valores inferiores a 5 UNT.

Os valores encontrados de pH estão dentro da faixa esperada para uma água que passou por uma estação de tratamento, respaldando as validações realizadas com o pHmetro da UFCG. O pH das águas naturais está geralmente compreendido entre a faixa de 6 a 8 . Entretanto, esse índice pode sofrer variações quando há contato da água com materiais orgânicos, seres vivos fotossintetizantes, rochas, gases atmosféricos e despejos domésticos ou industriais, por exemplo, o que não se aplicaria para o monitoramento em um sistema fechado.

Caso a água estivesse contaminada com a presença de microrganismos vivos, poderia ocorrer alterações no pH da água devido ao consumo ou produção de dióxido de carbono, o que resultaria na produção de ácidos orgânicos fracos (CÂNDIDO et al., 2015). Todavia, o período de 10 dias para avaliação do sistema não seria suficiente para que tais alterações se manifestassem de forma significativa, sendo para isso necessários testes em períodos maiores de tempo. 
A grande maioria dos medidores de multiparâmetros da água vendidos no mercado contam também com o monitoramento da temperatura da água. Para verificar possíveis alterações de pH ou turbidez com a variação da temperatura da água ao longo de um dia, optou-se também pelo acréscimo de um sensor de temperatura ao sistema deste estudo.

Diante dos elevados custos de muitos dos sistemas de automação que são comercializados, algumas tecnologias têm se tornado aliadas no desenvolvimento de sensores inteligentes de baixo custo, tais como o armazenamento de dados em nuvens, o uso de pequenas baterias para alimentação dos hardwares e também as redes de transmissão de dados com boas velocidades, obtidas via operadoras telefônicas que fornecem serviços de internet com 4G (ZHANG et al., 2019).

Garantir o acesso contínuo à internet, de forma que o monitoramento ocorra em tempo real e as notificações sobre a qualidade da água sejam entregues, é de grande importância para o sucesso deste sistema. Para isso, recomenda-se investigar a intensidade do sinal de internet que é obtido por meio de chip de telefonia móvel ou de uma rede Wi-fi disponível, comparando ambos os resultados.

A utilização do microcontrolador Node MCU para conexão à uma rede Wi-Fi mostrou-se mais estável do que uma conexão via chip de telefonia móvel quando submetidos à testes. Conectado à rede sem fio do laboratório de Saneamento da UFCG, o microcontrolador não apresentou sinais de instabilidade no envio de dados à plataforma ThingSpeak. A opção por utilizar uma conexão wireless, quando esta existir, também implica na redução dos custos de investimento em manutenção de uma rede de telefonia móvel. A Figura 12 mostra o sistema montado de duas formas distintas: uma com o módulo para conexão 3G/4G e outra com o módulo Wi-Fi.

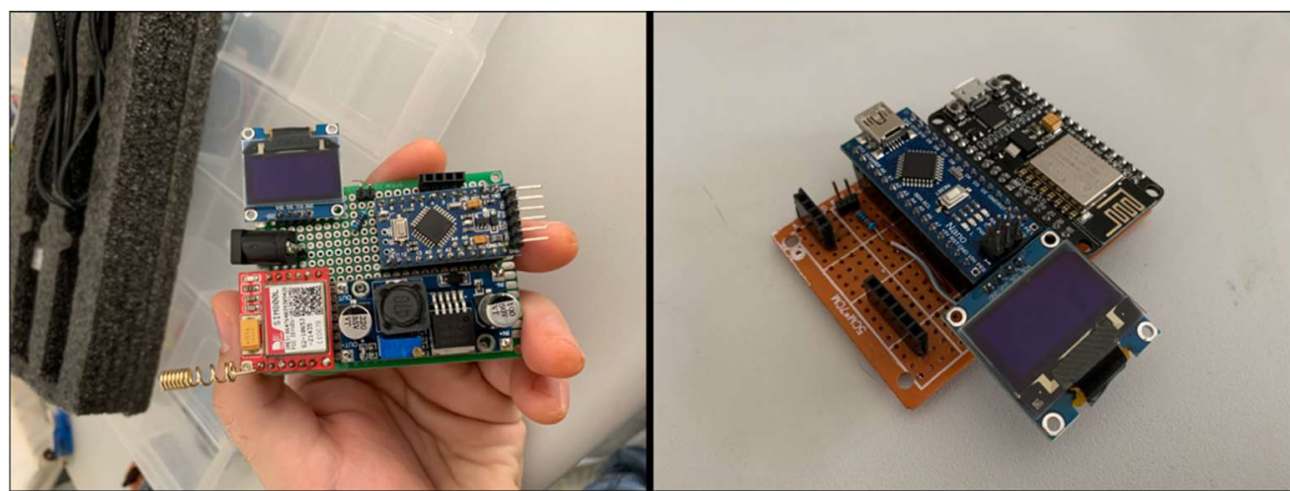

Figura 12: Comparativo entre o sistema com módulo para conexão 3G/4G e com módulo para conexão Wi-Fi.

Após os testes de durabilidade da bateria de $9 \mathrm{~V}$, observou-se por intermédio dos intervalos de envio dos dados à plataforma ThingSpeak, que o sistema durou apenas um dia e meio em pleno funcionamento. Também foi constatado que a eficiência máxima da bateria foi de oito horas. Após esse período, a tensão fornecida pela bateria foi menor do que 5V, que é a tensão adequada para o funcionamento dos sensores, acarretando em alterações nas leituras de pH e Turbidez. Apenas o sensor de temperatura, que independe da tensão fornecida pela bateria, manteve o envio de dados constantes mesmo durante o processamento de descarregamento da bateria.

Para a elaboração deste trabalho, o fornecimento de energia foi feito com uma fonte de $5 \mathrm{~V}$ ligada na 
tomada. Tal método de obtenção de energia, apesar de ter indicação de uso somente em ambientes controlados, ofertou plenas condições de funcionamento para um correto monitoramento da qualidade da água, sem nenhuma variação na tensão de entrada nos sensores.

Um sistema solar fotovoltaico para a alimentação de baterias é uma opção sugerida para trabalhos futuros de automações de sistemas de monitoramento da qualidade da água para comunidades rurais ou caminhões-pipa. Para isso, o correto dimensionamento das placas solares e das baterias se faria necessário. Placas solares e pequenas baterias recarregáveis para projetos de IoT podem ser encontradas com facilidade no mercado, sendo ideais para a elaboração de projetos de baixo custo.

Algumas adaptações se fizeram necessárias também na montagem dos circuitos para garantir um bom monitoramento da qualidade da água. Objetivando construir um sistema mais duradouro e resistente ao manuseio e até a eventuais impactos físicos, foi feita a tentativa de instalação do sistema utilizando-se uma placa de circuito impresso $(\mathrm{PCl})$ com soldagem artesanal. Porém, a alta taxa de retrabalho de soldagem quando era necessária alguma alteração no layout de circuitos do projeto, tornou a sua utilização pouco viável quando se tratava da criação de um protótipo do sistema, submetido a diversos testes que demandavam constantes alterações na disposição dos componentes do sistema. Além disso, eventuais problemas de curto-circuito, ocasionados por contatos indevidos entre diferentes trilhas condutoras, geravam alterações nas tensões recebidas pelos sensores. A montagem da PCI pode ser vista na Figura 13.

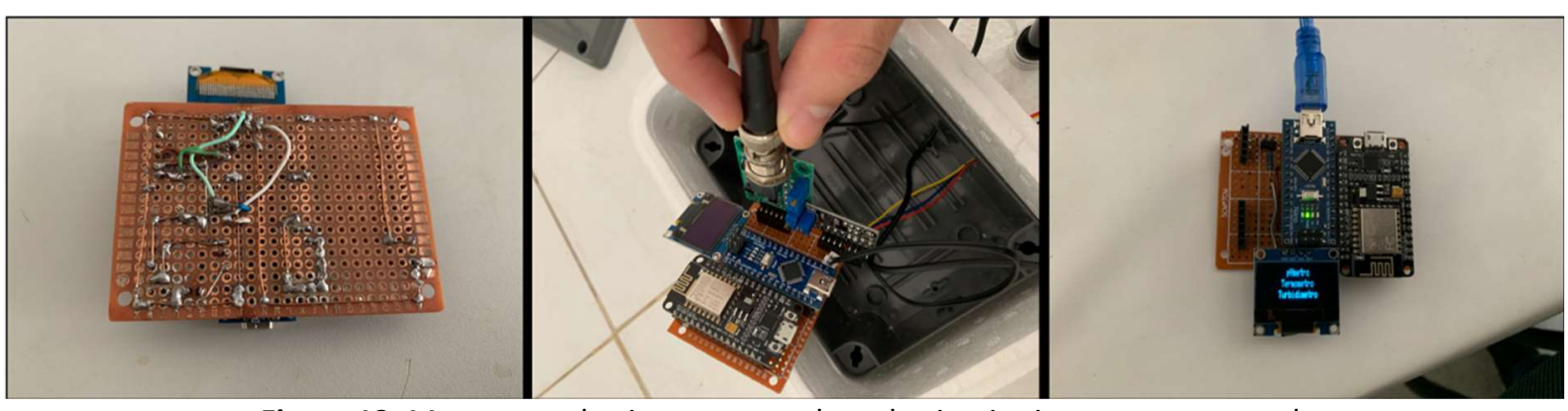

Figura 13: Montagem do sistema com placa de circuito impresso artesanal.

Como os sensores deste estudo funcionavam convertendo leituras de tensões para dados reais de $\mathrm{pH}$ e turbidez, qualquer variação de tensão poderia alterar significativamente os dados que estavam sendo medidos. Tendo isso em vista, optou-se por utilizar protoboards para a montagem do sistema, o que permitiu uma velocidade maior entre cada tentativa de montagem. Entretanto, essas tornaram o sistema de monitoramento suscetível a falhas caso algum dos jumpers fosse desconectado.

As extremidades dos sensores utilizados neste estudo necessitavam estar submersos na água, para que as leituras de $\mathrm{pH}$, turbidez e temperatura ocorressem de forma correta. Os comprimentos pequenos dos cabeamentos que ligavam os sensores ao microntrolador Arduino, limitaram a distância entre os sensores e a caixa de proteção, que armazenava os componentes eletrônicos do sistema.

Diante disso, optou-se pela utilização de uma caixa de proteção para possibilitar a flutuação do sistema. Uma caixa de isopor, com dimensões ligeiramente superiores as dimensões da caixa de proteção, foi utilizada como solução para fazer o sistema flutuar. Para permitir a passagem dos cabos que ligavam os 
sensores ao microcontrolador Arduino, foram feitos três pequenos futuros na caixa de proteção. A figura 14 mostra o protótipo do sistema montado.

O abastecimento público se dá por redes de distribuição e também através de veículos transportadores, de sistemas de captação de água de chuva em cisternas, por captação direta de recursos hídricos com auxílio de baldes, através da coleta em poços, entre outros. Em todas essas formas de acesso, a vigilância da qualidade da água se faz necessária para garantir boa qualidade de vida aos seus usuários (MACHADO et al., 2016).

O uso de sistemas automatizados para monitoramento da qualidade da água de cisternas e poços de comunidades rurais, apresenta-se como alternativa às coletas de amostras de água de forma convencional. A economia de tempo, recursos financeiros e de mobilização de pessoal em campo, justificaria a implantação de sensores e dispositivos para transmissão de dados de forma remota. De posse dos valores medidos, os órgãos de vigilância poderiam fazer ações reparatórias na qualidade da água de forma mais pontual, evitando-se maiores esforços de deslocamento e investigação. A Figura 15 mostra um esquema de monitoramento da qualidade da água para cisternas rurais.
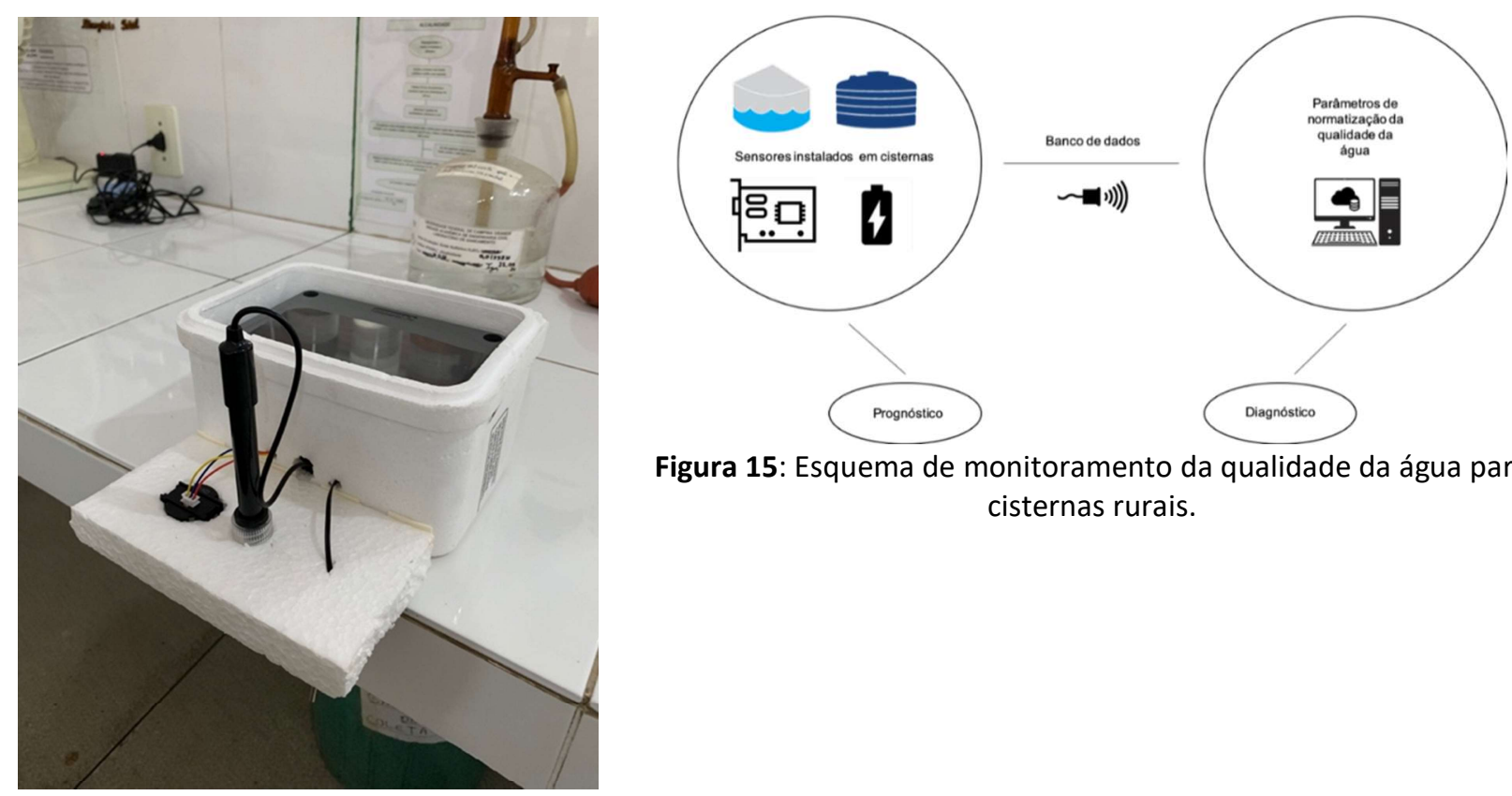

Figura 15: Esquema de monitoramento da qualidade da água para cisternas rurais.

Figura 14: Protótipo do sistema de monitoramento da qualidade da água.

Além de ser usado em águas de cisternas e poços, o sistema de monitoramento automatizado e em tempo real da qualidade da água em caminhões-pipa, contribuiria para elevar a segurança do usuário final ao informar aos responsáveis pelo monitoramento remoto sobre eventuais alterações na qualidade da qualidade da água, possibilitando que ações reparatórias fossem tomadas. Medições inteligentes via sensores acoplados nos tanques de armazenamento de água desses veículos, com o auxílio de dispositivos de transmissão de dados 3G/4G, poderiam auxiliar no controle de eventuais alterações nos parâmetros de potabilidade. As dificuldades de captação de sinal de internet em localidades remotas poderiam ser supridas pelo uso de cartões de memória, que armazenariam o histórico de monitoramento ao longo do dia para posteriores análises. 
A avaliação da qualidade da água por sensores de baixo custo ainda enfrenta alguns desafios, como a programação para leitura de baixa potência, a transferência automática de dados, principalmente em áreas que contam com sinais fracos de internet, alternativas de fornecimento de energia, durabilidade dos componentes e portabilidade dos dispositivos de monitoramento. Ainda assim, o desenvolvimento de sistemas para monitoramento remoto da água de consumo humano pode ser promissor ao beneficiar comunidades de zonas rurais e de difícil acesso, contribuindo para o melhoramento da qualidade de vida dessas populações (SRIVASTAVA et al., 2018). O custo do sistema desenvolvido neste estudo foi de R\$430,46, cujo detalhamento está descrito na Tabela 2.

Tabela 2: Valores dos materiais utilizados.

\begin{tabular}{|l|l|l|l|}
\hline Materiais & Quantidade & Preço Unitário (R\$) & Preço Total (R\$) \\
\hline Node MCU & 1 & 27,90 & 27,90 \\
\hline Arduino Nano & 1 & 22,90 & 22,90 \\
\hline Sensor de pH & 1 & 121,69 & 121,69 \\
\hline Sensor de Turbidez & 1 & 133,98 & 133,98 \\
\hline Sensor de temperatura & 1 & 14,90 & 14,90 \\
\hline Resistor 10K Ohm & 1 & 1,00 & 1,00 \\
\hline Potenciômetro 1K Ohm & 1 & 2,00 & 2,00 \\
\hline Display OLED & 1 & 22,90 & 22,90 \\
\hline Fonte 5V & 1 & 15,99 & 15,99 \\
\hline Cabo mini USB & 9,90 & 9,90 \\
\hline Protoboard & 1 & 10,90 & 21,80 \\
\hline Jumpers & 2 & 0,30 & 4,50 \\
\hline Caixa de proteção & 15 & 19,00 & 19,00 \\
\hline Isopor & 1 & 12,00 & 12,00 \\
\hline Custo Total & 1 & & \\
\hline
\end{tabular}

Os equipamentos listados na Tabela 3 possuem propostas similares à deste trabalho, objetivando o monitoramento de parâmetros qualitativos da água. Como diferencial, o sistema de monitoramento da qualidade da água desenvolvido neste trabalho permitiu a captação e transmissão dos valores lidos via internet em tempo real, além de enviar notificações personalizadas à computadores ou dispositivos móveis sobre o seu funcionamento. Os valores dos equipamentos portáteis de monitoramento da água citados na Tabela 3 foram consultados em julho de 2020. A partir dos valores apresentados, é possível identificar que o custo total do sistema desenvolvido neste trabalho representa um valor de $35 \%$ do custo do equipamento de monitoramento mais barato vendido no mercado.

Tabela 3: Valores médios de equipamentos similares fornecidos no mercado.

\begin{tabular}{|c|c|c|c|c|c|c|c|}
\hline \multirow{2}{*}{ Equipamento } & \multicolumn{6}{|c|}{ Sensores } & \multirow{2}{*}{ Valor } \\
\hline & $\mathrm{pH}$ & CE & Temp. & OD & STD & Salin. & \\
\hline Medidor Multiparâmetro PH-026 Impac & $\mathrm{x}$ & $\mathrm{x}$ & $\mathrm{x}$ & & & & $\mathrm{R} \$ 1.247,62$ \\
\hline Medidor Multiparâmetro WA-2015 Lutron & $x$ & $\mathrm{x}$ & $\mathrm{x}$ & $\mathrm{x}$ & & & $\mathrm{R} \$ 4.742,74$ \\
\hline Medidor Multiparâmetro HI9813-5 Hanna & $\mathrm{x}$ & $x$ & $\mathrm{x}$ & & $x$ & & $\mathrm{R} \$ 1.433,30$ \\
\hline Medidor Multiparâmetro HI98129 Hanna & $x$ & $x$ & $x$ & & $x$ & & $\mathrm{R} \$ 1.230,12$ \\
\hline Medidor Multiparâmetro Combo 5 & $x$ & $x$ & $\mathrm{x}$ & & $x$ & $\mathrm{x}$ & $\mathrm{R} \$ 1.515,72$ \\
\hline Medidor Multiparâmetro AK88 Akso & $\mathrm{x}$ & $\mathrm{x}$ & $\mathrm{x}$ & $\mathrm{x}$ & & & $\mathrm{R} \$ 2.840,00$ \\
\hline Medidor Multiparâmetro PTC-100 Instrutherm & $\mathrm{x}$ & $\mathrm{x}$ & $x$ & & & & $\mathrm{R} \$ 1.228,19$ \\
\hline Legenda & \multicolumn{7}{|c|}{$\begin{array}{l}\text { pH: Potencial Hidrogeniônico; CE: Condutividade Elétrica; } \\
\text { Temp.: Temperatura da Água; OD: Oxigênio Dissolvido; } \\
\text { STD: Sólidos Totais Dissolivos; Salin.: Salinidade. }\end{array}$} \\
\hline
\end{tabular}




\section{CONCLUSÕES}

Os resultados deste estudo comprovaram a eficiência do sistema de baixo custo para monitoramento remoto da qualidade de água. Os resultados das medições de $\mathrm{pH}$, turbidez e temperatura nesse sistema se mostraram coerentes com os valores das leituras feitos no pHmetro e turbidímetro do laboratório de Saneamento da UFCG. A plataforma ThingSpeak também se mostrou uma excelente alternativa gratuita para o recebimento dos dados.

Tomando-se como referência os equipamentos do laboratório de saneamento da UFCG, o sistema desenvolvido apresentou erros de 0,261\% para leituras de $\mathrm{pH}$ e 2,45\% para leituras de turbidez. A utilização de baterias para alimentação do sistema não foi bem-sucedida, optando-se então pela alimentação do sistema por uma tomada de 220V. Diante disso, é sugerido o estudo de formas alternativas para alimentação do sistema, como o uso de placas fotovoltaicas para alimentação de baterias. A montagem dos circuitos em protoboards tornou o sistema frágil a movimentações e eventuais impactos que viessem a acontecer em situações de campo, sendo recomendado o uso de placas de circuito impresso para aumentar a sua vida útil.

O baixo custo para a criação do sistema desenvolvido neste trabalho mostra-se favorável para a aplicação do monitoramento remoto em áreas de difíceis acessos, de forma a ampliar a segurança no consumo da água em tais localidades. O sistema desenvolvido neste trabalho teve um custo total de $\mathrm{R} \$ 430,46$, valor inferior aos preços de equipamentos semelhantes encontrados no mercado. Como diferencial com relação aos medidores de multiparâmetros convencionais, o sistema proposto permite conexão à internet para envio de dados e de notificações em tempo real, permitindo o monitoramento da qualidade da água por meio de celulares, tablets ou computadores, reduzindo assim a necessidade de deslocamento de equipes de campo.

\section{REFERÊNCIAS}

ABOUBARAKA, A. E.; ABOELFETOH, E. F.; EBEID, EL-Z. M. Coagulation effectiveness of graphene oxide for the removal of turbidity from raw surface water. Chemosphere, v.181, p.738-746, 2017. DOI: http://doi.org/10.1016/j.chemosphere.2017.04.137

BRASIL. Portaria de consolidação n. 5, de 28 de setembro de 2017. Brasília: DOU, 2017.

CÂNDIDO, T. S.; MIRANDA, J. H.; ABREU, M. V. S.; QUARTAROLI, L.. Avaliação da qualidade da água por meio de parâmetros físico-químicos e a influência do uso e ocupação do solo. Revista Científica Univiçosa, Viçosa, v.7, n.1, p.329-334, 2015.

CHAGAS, D. S.. Relação entre concentração de sólidos suspensos e turbidez da água medida com sensor de retroespalhamento óptico. Tese (Doutorado em Engenharia Agrícola) - Universidade Federal do Recôncavo da Bahia, Cruz das Almas, 2015.

GOMES, U. A. F.; HELLER, L.. Acesso à água proporcionado pelo Programa de Formação e Mobilização Social para Convivência com o Semiárido: Um Milhão de Cisternas Rurais. Engenharia Sanitária e Ambiental, Rio de Janeiro, v.21, n.3, p.623-633, 2016. DOI:

http://doi.org/10.1590/S1413-41522016128417

LAMBERTI, G. G.. Solubilização de matéria orgânica e nutrientes do lodo algáceo de uma lagoa de estabilização através de processos hidrolíticos alcalino e térmico. Dissertação (Mestrado em Engenharia e Desenvolvimento Sustentável) - Universidade Federal do Espírito Santo, Vitória, 2017.

MACHADO, A. V. M.; SANTOS, J. A. N.; NOGUEIRA, L. T.; NOGUEIRA, M. T.; OLIVEIRA, P. A. D.. Acesso ao abastecimento de água em comunidades rurais: o desafio de garantir os direitos humanos à água. In: CONGRESSO NACIONAL DE EXCELÊNCIA EM GESTÃO, 12. Anais. Rio de Janeiro: Inovarse, 2016.

MUOIO, R.; CARETTI, C.; ROSSI, L.; SANTIANNI, D.; LUBELLO, C.. Water safety plans and risk assessment: A novel procedure applied to treated water turbidity and gastrointestinal diseases. International Journal of Hygiene and Environmental Health, v.223, n.1, p.281-288, 2020. DOI: http://doi.org/10.1016/j.ijheh.2019.07.008

PASIKA, S.; GANDLA, S. T.. Smart water quality monitoring 
system with cost-effective using loT. Heliyon, v.6, n.7, p.1-9, 2020. DOI: http://doi.org/10.1016/i.heliyon.2020.e04096

ROCHA, R. S.; ITO, A. H.; LAUTENSCHLAGER, S. R.. Uso de sensores inteligentes com transmissão sem fio para monitoramento de cloro residual na rede de distribuição de água de Jussara-PR. In: CONGRESSO NACIONAL DE SANEAMENTO DA ASSAMAE: ALTERNATIVAS DE FINANCIAMENTOS PARA O SANEAMENTO PÚBLICO, 48. Anais. Fortaleza: ASSAMAE, 2018.

SALLA, M. R.; SÁ, E.; FERREIRA, P. A. S. C.; MELO, N. A.. Relação entre saneamento básico e saúde pública em Bissau, Guiné-Bissau. Saúde e Sociedade, v.28, n.4, p.284-296, 2019. DOI: http://doi.org/10.1590/s0104-12902019180705

SRIVASTAVA, S.; VADDADI, S.; SADISTAP, S.. Smartphonebased System for water quality analysis. Applied Water
Science, v.8, n.5, p.1-13, 2018. DOI:

http://doi.org/10.1007/s13201-018-0780-0

STEVENSON, M.; BRAVO, C.. Advanced turbidity prediction for operational water supply planning. Decision Support Systems, v.119, p.72-84, 2019. DOI: http://doi.org/10.1016/j.dss.2019.02.009

VENTURA, K. S.; VAZ FILHO, P.; NASCIMENTO, S. G.. Plano de segurança da água implementado na estação de tratamento de água de Guaraú, em São Paulo. Engenharia Sanitária e Ambiental, Rio de Janeiro, v.24, n.1, p.109-119, 2019. DOI: http://doi.org/10.1590/S1413-41522019169881

ZHANG, D.; HEERY, B.; O'NEIL, M.; LITTLE, S.; O'CONNOR, N. E.; REGAN, F.. A Low-Cost Smart Sensor Network for Catchment Monitoring. Sensors, v.19, n.10, p.2278-2300, 2019. DOI: http://doi.org/10.3390/s19102278

A CBPC - Companhia Brasileira de Produção Científica (CNPJ: 11.221.422/0001-03) detém os direitos materiais desta publicação. Os direitos referem-se à publicação do trabalho em qualquer parte do mundo, incluindo os direitos às renovações, expansões e disseminações da contribuição, bem como outros direitos subsidiários. Todos os trabalhos publicados eletronicamente poderão posteriormente ser publicados em coletâneas impressas sob coordenação da Sustenere Publishing, da Companhia Brasileira de Produção Científica e seus parceiros autorizados. Os (as) autores (as) preservam os direitos autorais, mas não têm permissão para a publicação da contribuição em outro meio, impresso ou digital, em português ou em tradução. 